\title{
A|P $\begin{aligned} & \text { The Journal of } \\ & \text { Chemical Physics }\end{aligned}$
}

Femtosecond real-time probing of reactions. II. The dissociation reaction of ICN

Marcos Dantus, Mark J. Rosker, and Ahmed H. Zewail

Citation: The Journal of Chemical Physics 89, 6128 (1988); doi: 10.1063/1.455428

View online: http://dx.doi.org/10.1063/1.455428

View Table of Contents: http://scitation.aip.org/content/aip/journal/jcp/89/10?ver=pdfcov

Published by the AIP Publishing

Articles you may be interested in

Femtosecond real-time probing of reactions. V. The reaction of $\mathrm{IHgl}$

J. Chem. Phys. 91, 7437 (1989); 10.1063/1.457267

Femtosecond real-time probing of reactions. IV. The reactions of alkali halides

J. Chem. Phys. 91, 7415 (1989); 10.1063/1.457266

Femtosecond real-time probing of reactions. III. Inversion to the potential from femtosecond transition-state spectroscopy experiments

J. Chem. Phys. 90, 829 (1989); 10.1063/1.456108

Femtosecond real-time probing of reactions. I. The technique

J. Chem. Phys. 89, 6113 (1988); 10.1063/1.455427

Real-time femtosecond probing of "transition states" in chemical reactions

J. Chem. Phys. 87, 2395 (1987); 10.1063/1.453122

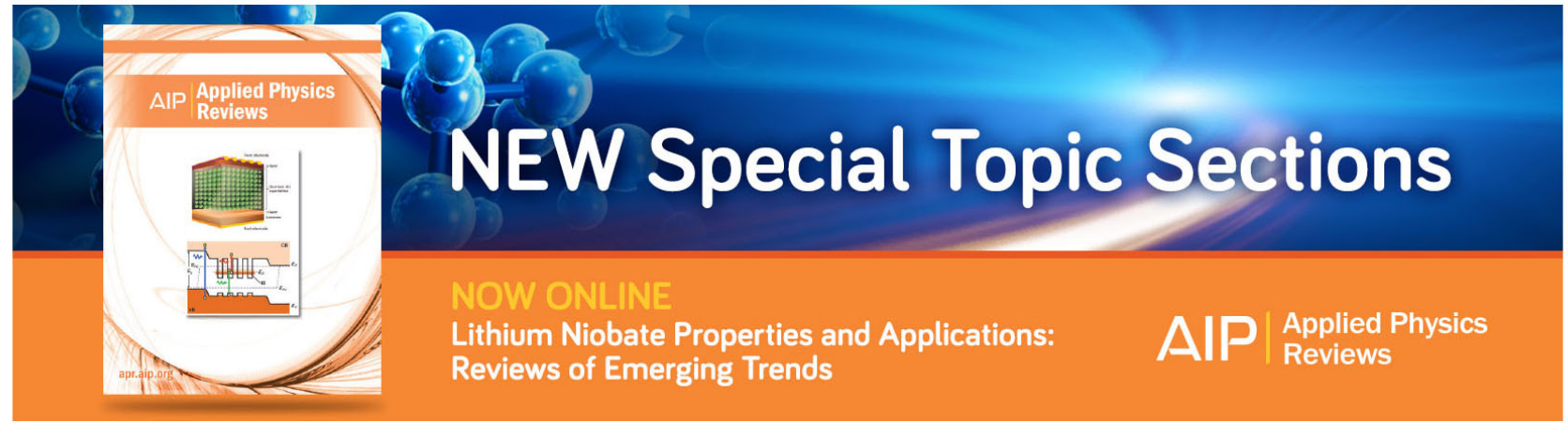




\title{
Femtosecond real-time probing of reactions. II. The dissociation reaction of ICN
}

\author{
Marcos Dantus, Mark J. Rosker, and Ahmed H. Zewaila) \\ Arthur Amos Noyes Laboratory of Chemical Physics, California Institute of Technology, Pasadena, \\ California 91125
}

(Received 12 July 1988; accepted 11 August 1988)

\begin{abstract}
Experimental results obtained for the dissociation reaction
$\mathrm{ICN}^{*} \rightarrow[\mathrm{I} \cdots \mathrm{CN}]^{\ddagger *} \rightarrow \mathrm{I}+\mathrm{CN}$ using femtosecond transition-state spectroscopy (FTS) are presented. The process of the I-CN bond breaking is clocked, and the transition states of the reaction are observed in real time. From the clocking experiments, a "dissociation" time of $205 \pm 30 \mathrm{fs}$ was measured and was related to the length scale of the potential. The transition states live for only $\sim 50 \mathrm{fs}$ or less, and from the observed transients we deduce some characteristics of the relevant potential energy surfaces (PES). These FTS experiments are discussed in relation to both classical and quantum mechanical models of the dynamical motion, including features of the femtosecond coherence and alignment of fragments during recoil. The observations are related to the radial and angular properties of the PES.
\end{abstract}

\section{INTRODUCTION}

Femtosecond transition-state spectroscopy (FTS) has been described in the first paper of this series (I). ' Here, we present experimental results obtained with FTS for the dissociation reaction of $\mathrm{ICN}$ :

$$
\mathrm{ICN}^{*} \rightarrow[\mathrm{I} \cdots \mathrm{CN}]^{ \pm *} \rightarrow \mathrm{I}+\mathrm{CN} .
$$

In our first FTS studies of this reaction, ${ }^{2}$ we reported the observation of the transition states $[1 \cdots \mathrm{CN}]^{\ddagger *}$ and gave the time scale for the formation of the free- $\mathrm{CN}$ fragment. In this paper, an account of the earlier work is given and a more complete description of the dynamics is presented using the simple classical and quantum mechanical theories discussed in $\mathrm{I}$.

Studies of the dissociation reaction of ICN with FTS is attractive for several reasons. First, a wealth of information is known about the photodissociation of this linear (groundstate) molecule. Second, the fact that ICN is a triatomic molecule could allow us to more easily compare experiments with theory. Finally, the energy can be deposited in the I$\mathrm{CN}$ bond, and the dissociation coordinate is that of a quasidiatomic molecule. Nevertheless, there remains a number of unanswered questions about the dynamics of the ICN reaction, especially the shapes of the potential energy surfaces (PES) involved.

It has been established that excitation of ICN to the $\tilde{A}$ continuum $^{3}$ leads to dissociation via two channels ${ }^{4}$ :

$$
\begin{aligned}
\mathrm{ICN}+h v & \rightarrow \mathrm{I}\left({ }^{2} P_{3 / 2}\right)+\mathrm{CN}\left(X^{2} \Sigma^{+}\right) \\
& \rightarrow \mathrm{I}^{*}\left({ }^{2} P_{1 / 2}\right)+\mathrm{CN}\left(X^{2} \Sigma^{+}\right),
\end{aligned}
$$

producing iodine atoms either in the ground spin-orbit state I $\left({ }^{2} P_{3 / 2}\right)$, or in the excited state $I^{*}\left({ }^{2} P_{1 / 2}\right)$. For both channels (see Fig. 1), the CN fragment is produced in the ground electronic state; at least $99.9 \%$ of the $\mathrm{CN}$ fragment was found in its $\left(X^{2} \Sigma^{+}\right)$state. $^{5}$

\footnotetext{
a) John Simon Guggenheim Foundation Fellow.
}

In contrast to the cold electronic and vibrational excitation of the $\mathrm{CN}$, the rotational excitation (e.g., for the $266 \mathrm{~nm}$ excitation, near the peak of the $\widetilde{A}$ continuum) is rich and shows two distributions corresponding to the two channels. Photofragment angular distribution measurements, ${ }^{6}$ product state distribution, ${ }^{7}$ product yield of the $\mathrm{I}$ and $\mathrm{I}^{*}$ channels, ${ }^{8}$ sub-Doppler LIF measurements, ${ }^{9}$ and spatial anisotropy and alignment experiments ${ }^{10}$ have all been invoked to explain the dynamics of the different dissociation channels. On the basis of symmetry correlation diagrams it has been concluded $^{\text {" }}$ that the state which correlates with the I $+\mathrm{CN}$ channel is bent, while that which correlates with the $I^{*}+\mathrm{CN}$ channel is linear. If the molecule is bent, because of the torque on the $\mathrm{CN}$ we expect a high degree of rotational excitation in the $\mathrm{CN}$ photofragment distribution. As shown recently, ${ }^{9}$ for the $\mathrm{I}+\mathrm{CN}$ channel, high rotational quantum numbers of the $\mathrm{CN}\left(N^{\prime \prime}\right)$ are selectively produced, whereas for the $\mathrm{I}^{*}+\mathrm{CN}$ channel the distribution peaks sharply at low $N^{\prime \prime}$ (low rotational energy).

An interesting question relating to the dynamics is the following: After absorption are these two channels independently produced? Or is the absorption to one state (linear), which by curve crossing leads to two channels? Nadler et al. ${ }^{9}$ suggested that only one state is involved in absorption, and that this absorption is to a linear state having predominantly parallel character. This linear state(s), which correlates with $I^{*}+\mathrm{CN}$, crosses a bent state that produces $\mathrm{I}+\mathrm{CN}$. Zare's group, ${ }^{10}$ by measuring the alignment of the photofragment, concluded that the dynamics of the curve crossing between linear and bent surfaces takes place in the FranckCondon region. Further support comes from the calculations by Goldfield et $a l .^{12}$ of the rotational distribution and the yield of $I$ and $I^{*}$, using nonadiabatic interactions between the ICN surfaces. ${ }^{13}$

Our first subpicosecond ${ }^{14}$ and FTS $^{2}$ experiments on ICN, as well as all experiments described here (except for some at $285 \mathrm{~nm}$ ), were made at $306 \mathrm{~nm}$. At or near this wavelength, which is much to the red of the absorption peak and corresponds to a total available energy of $6000-7000$ 


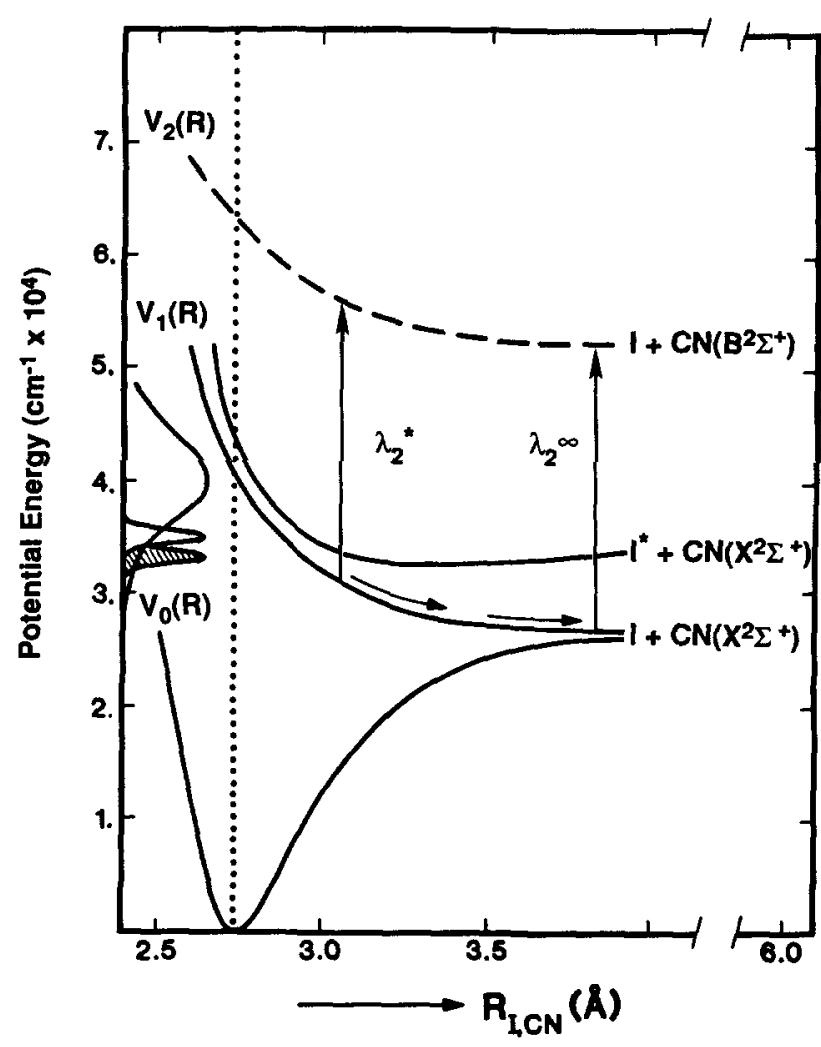

FIG. 1. Schematic of FTS and the potential energy surfaces of ICN. The pump pulse causes a vertical transition from $V_{0}$ to $V_{1}$, and the subsequent motion on this surface is indicated. The transition state is probed by $\lambda_{2}^{*}$, or the final state by $\lambda_{2}^{\infty}$, which are shown by the respective arrows from $V_{1}$ to $V_{2}$. The absorption spectrum of ICN and the pump energies used are sketched on the left. Note that the difference between $\lambda_{2}^{*}$ and $\lambda_{2}^{*}$ is not to scale, and that the van der Waals wells are not shown.

$\mathrm{cm}^{-1}$ above dissociation, only the $\mathrm{I}+\mathrm{CN}$ channel is produced, ${ }^{15}$ which simplifies the problem. (The spin-orbit splitting of iodine is $7600 \mathrm{~cm}^{-1}$.) For these wavelengths, the rotational distributions are known, and they peak around $N^{\prime \prime} \sim 25 .^{15}$ Simple kinematics can actually account ${ }^{16}$ for these rotational distributions at the energies of interest for this "direct" (i.e., < 1 ps) dissociation.

In the past, the source ${ }^{17}$ of information on the (average) lifetime $\langle\tau\rangle$ of dissociation came from measurements of angular distribution ( $\mathrm{s}$ ) and from the calculated average rotational period $\langle\omega\rangle$ of the parent molecule, as illustrated for the case of ICN by Ling and Wilson. ${ }^{6}$ Using the spatial anisotropy parameter $\beta^{17.18}$ one may estimate $\langle\tau\rangle$. For example, for a parallel-type transition and for recoil along the internuclear axis:

$$
\beta=2 \frac{1+(\langle\omega\rangle\langle\tau\rangle)^{2}}{1+4(\langle\omega\rangle\langle\tau\rangle)^{2}}
$$

Knowing $\beta$ and computing $\langle\omega\rangle\left(\sim 1 \times 10^{12} \mathrm{rad} \mathrm{s}^{-1}\right.$ for ICN at $300 \mathrm{~K}),{ }^{9}$ one deduces $\langle\tau\rangle$. This clever idea uses the rotation of the molecule as an average clock; if the molecule dissociates before it rotates then the anisotropy is very high. For ICN, $\langle\tau\rangle$ is estimated to be a few hundred femtoseconds.
There are several points, however, that must be considered. First, coupling between surfaces and/or changes in recoil direction make interpretations less straightforward. There is also the symmetry factor ${ }^{19}$ in $\beta$ which must be known. In applying Eq. (3) to ICN, we note the following: The rotational period $\langle\omega\rangle$ should depend on the temperature and, accordingly, $\beta$ should generally change with temperature if $\langle\tau\rangle$ is the same. For ICN, the angular distribution was found to be the same for room temperature $(300 \mathrm{~K})$ photodissociation as it was for supersonically cooled $(\sim 10$ K) ICN. ${ }^{20}$ The period $\langle\omega\rangle$ changes in these two experiments by a factor of 5 , since the most probable $J_{\mathrm{ICN}}$ are 31 and 6 , respectively, at the two temperatures $(J \hbar=I \omega)$. Either $\langle\tau\rangle$ does change or the dynamics of the dissociation are independent of the parent rotation. However, in the former instance if $\langle\omega\rangle\langle\tau\rangle \ll 1$, as is the case here, then Eq. (3) is not very sensitive to changes in either $\langle\omega\rangle$ or $\langle\tau\rangle$. Another point is that slight differences in the magnitude of $\beta$ have been attributed in the literature to changes in the direction of the velocity vector in the recoil process. As discussed by Zare, ${ }^{10}$ in the case of ICN this bending during recoil may change the magnitude of the $\mathrm{CN}$ rotational angular momentum but will not change its direction ( $N$ angular momentum remains opposite to $L$, orbital angular momentum, and thus perpendicular to the ICN plane). Accordingly, while $\beta$ may decrease, the angular momentum alignment should remain constant, which is in contradiction with the alignment experiments of O'Halloran et al. ${ }^{10}$ Therefore, it is of interest to follow the real-time femtosecond dynamics of this dissociation reaction by observing the transition states and the development of the free fragments.

Since the recoil velocity for the reaction at energies of interest is typically $\sim 0.025 \AA \mathrm{As}^{-1}$, the time resolution of these FTS experiments allows us to view, with good resolution, a few angstroms of recoil. Also, on this time scale we can study the loss of alignment and the development of the $\mathrm{CN}$ angular momenta as a function of time. Three types of experiments are reported here: (i) clocking of the process of I-CN bond breaking and direct determination of $\tau$ for different recoil velocities; (ii) characterization of femtosecond transients for the transition states $[\mathrm{I} \cdots \mathrm{CN}]^{\ddagger *}$; and (iii) studies of recoil fragment coherence and angular momenta using polarized femtosecond pulse arrangements. The results are discussed in relation to the radial and angular parts of the PES and to the real-time dynamics that produce the I and $\mathrm{CN}$ fragments.

The organization of the paper is as follows: In Sec. II, we describe the experimental details specific to the FTS of ICN. This includes a discussion of a number of characterization and diagnostic experiments that we have performed. In Sec. III, we give the results and discuss the different types of experiments performed in relation to clocking, transitionstate characterization, femtosecond coherence and alignment, and the description of the PES. Our conclusions are summarized in Sec. IV.

\section{EXPERIMENTAL}

The experimental methodology of FTS was presented in paper I of this series. In this section, we provide specific 
details pertinent to the FTS experiments of the ICN reaction.

\section{A. FTS experiments}

As discussed in paper $I$, the femtosecond pulses were generated from a colliding pulse mode-locked ring dye laser (CPM), and were amplified by a Nd:YAG-pumped pulsed dye amplifier (PDA). The output pulses of this combination had $\sim 300 \mu \mathrm{J}$ energy and $20 \mathrm{~Hz}$ repetition rate.

Conversion of this light to the pump frequency was accomplished using either of two procedures. For most of our ICN experiments, the pump wavelength was chosen to be at $\sim 306 \mathrm{~nm}$, which could be conveniently produced via second harmonic generation of a portion of the PDA output. This was performed by focusing the light into a thin $(500 \mu \mathrm{m})$, phase-matched KD*P crystal. Because of the high peak intensity of the femtosecond pulses, the conversion efficiency into the second harmonic exceeded $10 \%$. The second pump generation procedure yielded femtosecond light centered near $285 \mathrm{~nm}$. This was accomplished by sum frequency mixing in a KD*P crystal of a portion of the PDA output with residual, synchronous $532 \mathrm{~nm}$ radiation from the YAG pump laser. The efficiency of this conversion was much lower, being limited by the onset of optical damage to the crystal by the nanosecond YAG pulses.

The probe pulses measured the absorption of the system at or off-resonance to the free- $\mathrm{CN}$ transitions near the bandhead at $388.5 \mathrm{~nm}$. In our experiments, the probe was generated by mixing together in $\mathrm{KD}^{*} \mathrm{P}$ the remainder of the PDA output with $1.06 \mu \mathrm{m}$ light from the YAG laser (see Fig. 2). The probe pulses were tunable over a limited range of wavelengths ( 387 to $396 \mathrm{~nm}$ ) by careful adjustment of the cavity elements of the CPM, specifically the positioning of the intracavity prisms and of the saturable absorber jet. (Adjustment of the phase-matching angle for the nonlinear mixing crystal was also required.)

The spectral resolution $(\Delta v)$ of the probe could be adjusted in order to observe the transition states of ICN. Because of the inverse relationship between spectral and temporal pulse width, however, as $\Delta v$ was made smaller, the temporal resolution $(\Delta t)$ become poorer. Care was exercised to insure that the pulses were chirp free (transform limited), so that $\Delta v \Delta t=0.3$ to 0.5 . However, the effect of nontransform limited pulses was also investigated.

The pump and probe beams, with proper attenuation and parallel or perpendicular polarization, were delayed in time relative to one another in a Michelson interferometer, and were collinearly recombined and focused into the reaction chamber. For these reported experiments, the chamber was a custom-made cell arrangement (see I). The ICN was kept at room temperature and the pressure was maintained at $0.1-0.3$ Torr. The materials used in the cell construction [Teflon, Delrin, stainless steel (304), and quartz] were tested for inertness. Laser-induced fluorescence (LIF) of the $\mathrm{CN}$ fragment was detected, and was collected at right angle to the direction of the pump/probe pulses through a $f / 1.5$ lens arrangement. To suppress scattered light, black Teflon baffling was invoked, and the entrance and exit optical win-

\section{Generation of Pump and Probe Pulses}

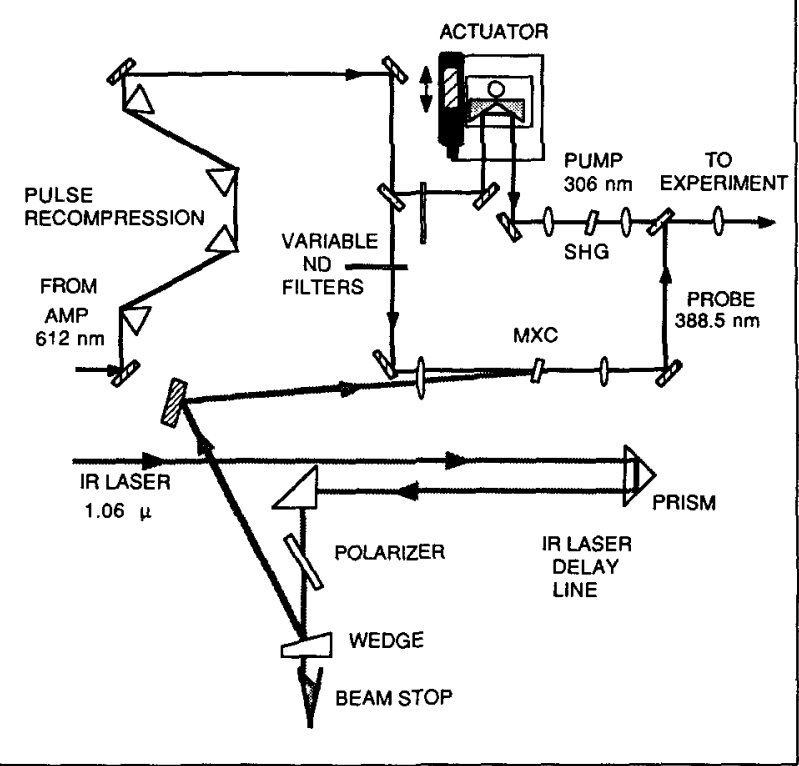

FIG. 2. Schematic of the pump/probe generation scheme. The output of the amplifier is first recompressed and then split into two equal beams. Each arm is then converted to the appropriate wavelength, by second harmonic generation (SHG) for the pump, and by sum frequency mixing (MXC) for the probe. The beams are then colinearly recombined. The delay time of the Michelson interferometer is provided by a precision actuator. (The $\sim 285$ $\mathrm{nm}$ pump generation technique, not shown, is similar to that of the probe generation.)

dows were oriented at Brewster's angle. Spurious light was also rejected by dispersing the LIF through a $0.34 \mathrm{~m}$ monochromator. A Hamamatsu R1527 photomultiplier tube (PMT) detected the LIF. A digital boxcar integrator averaged that portion of the PMT voltage which occurred within an adjustable temporal gate. The entire experiment was controlled by a microprocessor, which adjusted the optical delay line of the Michelson interferometer and acquired and numerically averaged together the boxcar output.

Characterization of the pump and probe pulses was considered crucial, and so for each data set, several additional measurements were made. First, the spectrum of the pump and the probe were each taken. Also, the cross correlation of the pump with the probe was measured, using the technique of difference frequency generation (DFG) in a nonlinear crystal $\left(\omega=\omega_{\text {pump }}-\omega_{\text {probe }}\right)$. The DFG light near $1.45 \mu \mathrm{m}$ was detected with a germanium photodiode. Further, we determined the overall temporal response function of our apparatus and the zero-of-time (defined by the temporal midpoint of the pump pulse).

Determination of the response function and the exact zero-of-time was critical to many of the experiments, and was accomplished as follows: The reaction chamber was constructed with an ionization electrode, which could be translated into the reaction region without movement of the cell or loss of vacuum. The cell also contained a second sample holder, containing $N, N$-diethylaniline (DEA). The re- 
sponse function measurement was made by evacuating the chamber of ICN and replacing it with DEA (at 0.3 Torr or less), positioning the ionization electrode in the reaction region, applying a dc voltage to the electrode, and measuring the resulting multiphoton ionization (MPI) current. Ionization of the DEA required both a pump and a probe photon, so that the MPI signal as a function of the time delay between the pump and probe gave the detection response function. ${ }^{14}$ We have also verified this by comparison of the DEA experiment results with the numerically integrated results of the cross correlation of the pump and probe. As discussed in paper I, the $t=0$ point was the time at which the MPI signal reached one-half of its limiting value. The response function measurement was made completely in situ, with no repositioning of the cell or adjustment of any optical elements. ${ }^{21}$

DEA was chosen for the calibration of the $t=0$ because the intermediate lifetime (aniline type) of the state reached by the pump pulse is long lived. Hence the signal will rise "instantaneously" with our pulse and will reach a plateau at long times, as observed experimentally. By instantaneous, we mean that it is as fast as any electronic transition response. We have considered, however, the possible existence of initial dephasing or redistribution, which would cause some delay in the MPI signal. However, in our experiments the DEA is excited very near the leading edge of absorption $\left(\lambda_{\max }=303 \mathrm{~nm}\right)^{22}$ so that IVR is on a very long time scale. ${ }^{23}$ Furthermore, rotational dephasing for this molecule at room temperature using the approach of Ref. 24 is $\approx 1 \mathrm{ps}$. All of these times (nanosecond to picosecond) are much longer than the time scale of our experiment. The precision of the $t=0$ calibration is fixed primarily by the experimental signal-to-noise ratio, but ultimately, the accuracy of the calibration will be limited by the response time of a molecule like DEA. Finally, it should be noted that since the cross section is $\sigma=1 \times 10^{-18} \mathrm{~cm}^{2}$ for DEA, ${ }^{22(b)}$ and the flux is $\Phi \sim 5 \times 10^{14}$ photons pulse ${ }^{-1} \mathrm{~cm}^{-2}$, then less than one in a thousand molecules is in the excited state. Hence, there is no saturation effect.

\section{B. Diagnostic experiments}

Numerous diagnostic experiments were performed in order to insure the proper treatment of the data. First, to establish the linearity of the signal with respect to the intensity of both the pump and the probe beams, the dependence of the LIF signal on the pump or probe energy was recorded. This measurement was performed under several experimental circumstances, including: (1) with an on-resonant probe $\left(\lambda_{2}=\lambda_{2}^{\infty}\right)$ and long time delay ( $\left.t \geqslant 1 \mathrm{ps}\right) ;(2)$ with an offresonant probe $\left(\lambda_{2}=\lambda \lambda_{2}^{*}\right)$ and long time delay; and (3) with an off-resonant probe and with the time delay set to maximize the FTS signal $\left(t=t^{*}\right)$. These results are plotted on a $\log -\log$ scale in Fig. 3. For each case, the data falls on a line with a slope near unity, which is evidence for the linearity of the FTS experiment (indicating that the ICN is photolyzed by a single pump photon, and that the fluorescence from the $\mathrm{CN}$ is induced by a single probe photon) as well as the detection apparatus. As a further test, FTS transients were acquired both on- and off-resonance over a wide range of pump and probe powers (factor of $\sim 10$ ). We have found no evi-
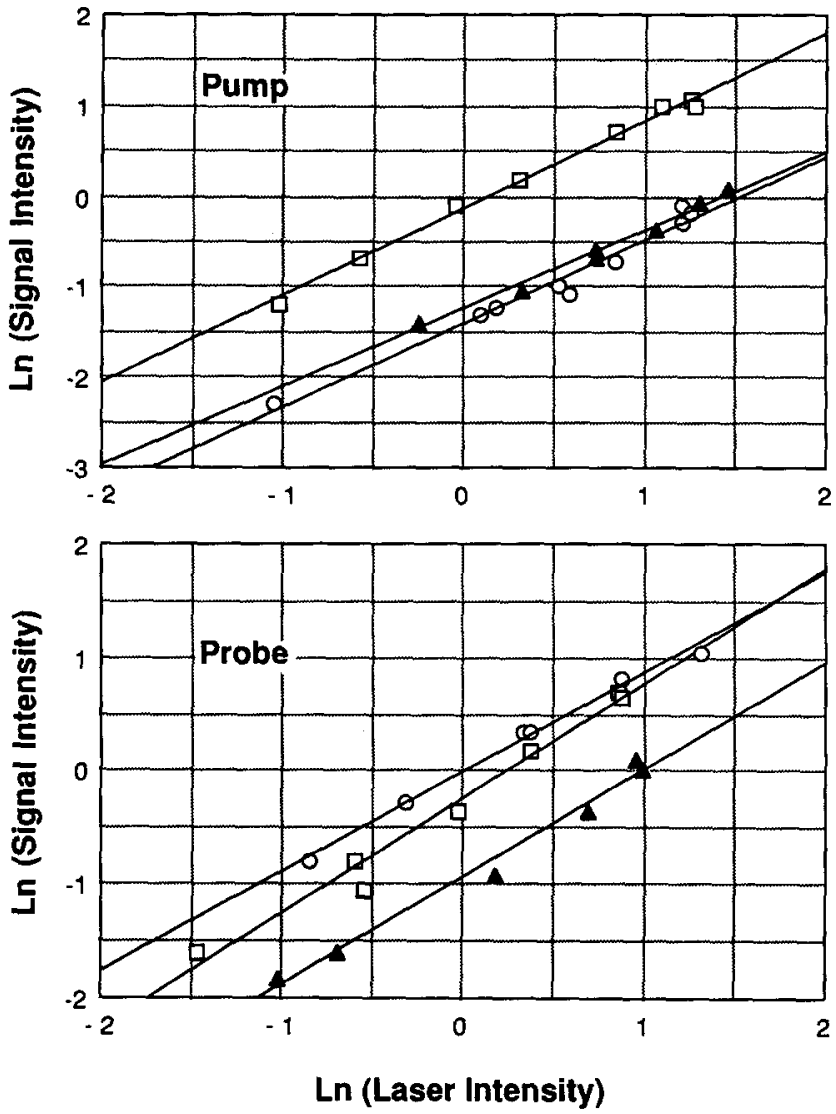

FIG. 3. Power dependence of the LIF signal. The observed LIF is plotted as a function of the pump or probe intensity on a log-log scale. Key: open squares: on-resonance transients; solid triangles and open circles: off-resonance transients for different time delays. The lines shown are best fits to the data. Note that, in all cases, the slopes of the lines are near unity.

dence of saturation in any of these measurements. These results are consistent with the sensitivity calculation presented in Sec. V A of paper I. Estimation of the total flux of photons in the measured LIF signal gives $10^{4}-10^{5}$ photons per pulse, which is consistent with these calculations (after taking into account various factors such as cross sections of the transitions, detector sensitivity, yields, etc.).

In order to characterize the fluorescence lifetime of the LIF signal of the $\mathrm{CN}$, we scanned the boxcar gate position and recorded the nanosecond decay of the $\mathrm{CN}$ radical. The recorded transient fit well to a single exponential decay, and the time constant was of the same order as previously reported values for the $\mathrm{CN}$ emission lifetime. ${ }^{25}$

Transients were also obtained for ICN pressures in the range $0.05-0.5$ Torr to check for any collisional effects. The only trend seen in this data was the expected decrease in the overall signal level as the pressure was reduced. The average collision time calculated at 0.2 Torr and room temperature is $\sim 0.1 \mu \mathrm{s}$. The effect of collisions on the dynamics of the reaction (especially the femtosecond dynamics) can be ignored.

The pump and the probe each contributed a background level to the observed data, which was independent of delay and superimposed on the delay-dependent FTS transient. A 
part of this "dc" level was determined to be caused by weak multiphoton absorption of the ICN by the pump light. This was easily reduced to $<10 \%$ of the peak signal by attenuation of the pump. The remaining contribution (also $\sim 10 \%$ of the peak level) was associated with scattered probe light. As already described, the techniques to discriminate against probe scatter included the use of baffles, Brewster windows, and temporal gating. Typical enhancements observed in these experiments when both the pump and the probe overlapped temporally were 10:1.

Since the scattered light persisted for only the detector response time $(<5 \mathrm{~ns})$, the gating of the boxcar was typically adjusted to open afterwards, and remain open for the rest of the fluorescence lifetime. The effect of inclusion in the observed FTS transients of the scattered probe light was studied by collecting transients with the following gate arrangements: (1) with the gate overlapping the early portion of the fluorescence (including the scattered probe light, if it existed); (2) with the gate opened only at the very end of the fluorescence; and (3) with an extremely wide gate, beginning well before the pump and probe pulses and extending for several fluorescence lifetimes. In each case, the shape of the FTS transient obtained was basically unchanged. As a further test, the scattered light level was increased by an order of magnitude by placing a card at the output Brewster window. Again, the measured FTS transient was unaffected (aside from the increase in the background level).

To check for polarization effects, we have verified that similar (but not identical) FTS transients were obtained for both parallel and perpendicularly polarized pump and probe beams. In all cases the probe beam polarization was oriented along the direction of the LIF collection, ${ }^{26}$ and the pump beam polarization was adjusted with the rotation of a zeroorder, half-wave retardation plate. The differences observed between these data sets will be discussed below.

The possibility of coherent interaction [see, e.g., Refs. 27 (a) -27 (c) ] between the pump and the probe was considered, and was ruled out. First, the transient shape is very sensitive to the probe wavelength within a range of a few nanometers; on-resonance $\left(\lambda_{2}^{\infty}\right)$ we observe only a delayed rise, and off-resonance $\left(\lambda_{2}^{*}\right)$ we see systematically a buildup and decay with a shift in delay time depending on $\lambda$. Second, the pump and probe are much different in wavelength, by $\sim 80 \mathrm{~nm}$. The very large difference in the respective wavelengths gives a coherence length for such an energy separation of only $1.4 \mu \mathrm{m}$, which is much smaller than the length of the interaction region (the confocal parameter of the optical pulses $=15 \mathrm{~mm}$ ). Equivalently, the coherence time is $5 \mathrm{fs}$, which is much shorter than the observation times. Because of the observed linearity of the signal (weak signal limit), the large difference between pump and probe wavelengths and the relatively small cross section $\left(\sim 4 \times 10^{-20} \mathrm{~cm}^{2}\right)$ at $\sim 306 \mathrm{~nm}$, we can also exclude the Stark shift produced by our pulses. [See, e.g., Ref. 27(d).]

Finally, the cyanogen iodide used in this study was prepared by vacuum sublimation of ICN (Aldrich, 95\%), which was repeated until the resulting material was completely colorless. The composition and purity of the compound was tested by mass spectroscopic methods. The $N, N$ - diethylaniline (Aldrich, 98\%) was fraction distilled until it became colorless and then was extensively outgassed.

\section{RESULTS AND DISCUSSION}

\section{A. Clocking experiment}

In the clocking experiments, described in paper $I$, the wavelength of the probe is tuned to be on-resonant with the final product state. In this case, $\lambda_{2}=\lambda_{2}^{\infty}=388.5 \mathrm{~nm}$, which is the transition wavelength of the bandhead for the $P$ branch of free-CN. Each data set collected consisted of an ICN transient, a DEA multiphoton ionization transient, and a probe spectrum.

Typical clocking results are presented in Fig. 4. The hollow circles in the figure are the experimental response function for the system, as determined by the DEA measurement. The zero-of-time is found from this data by the requirement that one-half of the limiting value is achieved at $t=0$. The solid squares in the figure show the FTS transient obtained with a $306 \mathrm{~nm}$ pump pulse. The femtosecond "time-of-flight" of the fragments is $\tau_{1 / 2}=205 \pm 30 \mathrm{fs}$. (The uncertainty here is an estimate from the scatter for several measurements of the observed $\tau_{1 / 2}$ values.) This value was obtained by least-squares fitting of the curves to the same function (solid lines). But independent of the fitting procedure, the delay of the free fragment is evident and measurable.
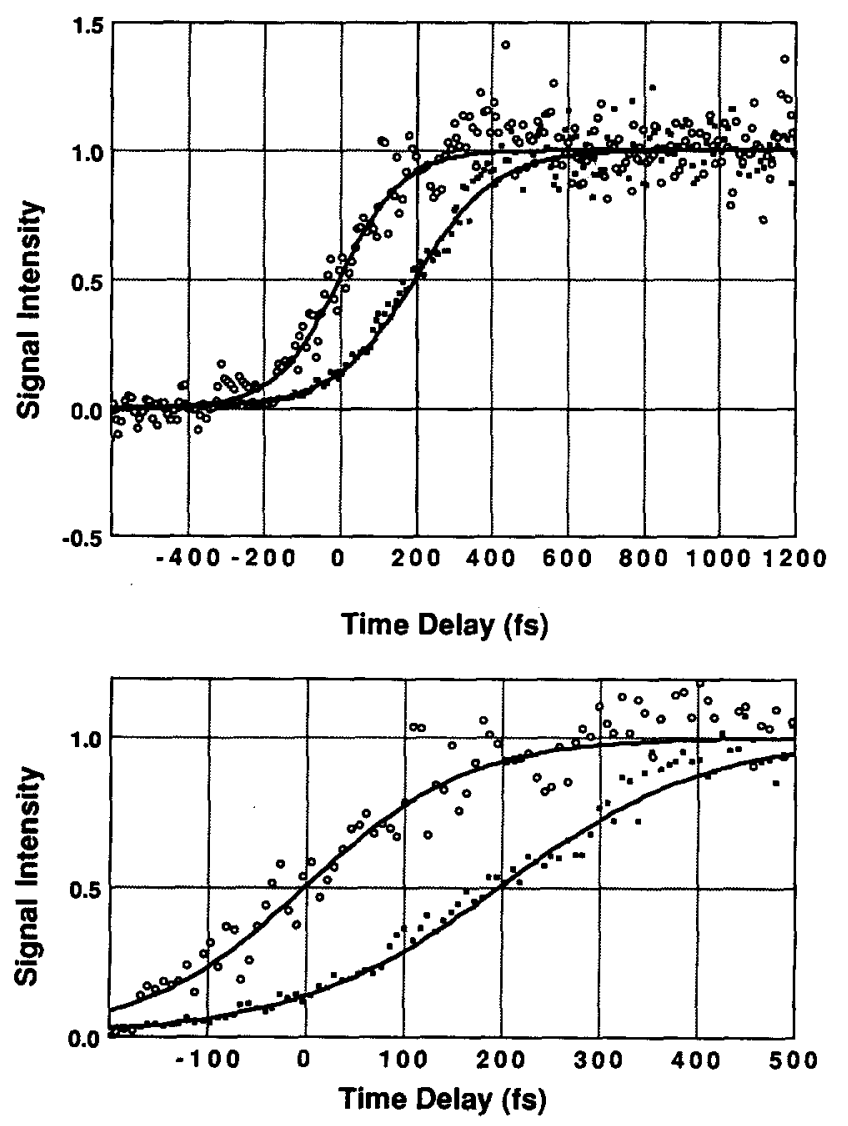

FIG. 4. Typical clocking experiment results with transform-limited pulses. The ICN transient (solid squares) in this case shows an observed delay of $\tau_{1 / 2}=205 \pm 30$ fs from the DEA transient (open circles). The lines are fits to the data. The figure at the bottom shows an expanded scale near $\tau=0$. 
Measurement of $\tau_{1 / 2}$ gives the time required to break the bond between the I and $\mathrm{CN}$ fragments. As discussed in paper I, the results of the clocking experiment are relatable to the shape of the PES, since $\tau_{1 / 2}$ is the length of time required to move with a recoil velocity $v(t)$ from the initially excited configuration at $R_{0}$ to the optically coupled region (OCR) of the probe. The terminal recoil velocity can be obtained from the translational energy in the center-of-mass frame: $E=1 / 2 \mu v^{2}$.

As a first approximation, suppose that the fragments moved on this path at the terminal recoil velocity, $v=0.026$ $\AA \mathrm{fs}^{-1} \cdot{ }^{28}$ At this velocity, the increased separation betweeen the fragments in $\tau_{1 / 2}$, from their initial separation, would be $5.3 \AA$. However, this value is essentially an upper bound, since the velocity of recoil begins at zero and changes with distance.

A more useful approach is to assume some functional form, e.g., an exponential repulsion between I and $\mathrm{CN}$, and to consider the reaction as a classical motion on this potential. This problem was considered in paper $I$, and it was shown, under the approximations made, that for the clocking experiment $\left(t^{*}=\infty\right)$ :

$$
\tau_{1 / 2}=\left(L_{1} / v\right) \ln (4 E / \gamma)
$$

where $\gamma$ is the half-width of the energy distribution of the probe pulse, and $E$ is the energy available above dissociation. Knowing $v, E$, and $\gamma$, we calculate from the measured $\tau_{1 / 2}$ that $L_{1}=0.8 \AA$. This length parameter is obtained assuming the one-dimensional potential, with no centrifugal term to account for the orbital angular momentum.

This brings to focus the problem of how to define the dissociation time. If it were defined as the time for the pair of fragments to reach a separation at which their interaction energy is negligible, the dissociation time would depend on the sensitivity with which that energy could be measured; an infinite time is required to reach infinite separation. A more invariant definition can be obtained through the following approach: Consider the difference between the time taken for the fragments to separate from $R_{0}$ to $R$ as they travel along the actual potential, minus the time which would have been required had the fragments traveled at the terminal velocity throughout. Then we may define the dissociation time $\tau_{d}$ as the limit of this difference as $R \rightarrow \infty$. On a repulsive surface, this leads to $\tau_{d}=\left(L_{1} / v\right) \ln 4 .{ }^{29}$ While this $\tau_{d}$ is well defined, it is obtainable only from a hypothetical experiment requiring the measurement of minute differences in long arrival times. See paper I, Fig. 14.

Equation (4) defines the physics of the problem more clearly: dissociation occurs when the fragment is spectroscopically identifiable as free (or nearly free) from the other fragment. Hence, the effect of $E, \gamma$, and $v$ is understood (see I). Tests of these dependences will be discussed below. The value of $L_{1}=0.8 \AA$ obtained from Eq. (4), it should be noted, represents the length at which the potential falls to $1 / e$ of its initial value; the distance at which the bond is "completely" broken is several times this value. As discussed in paper I, $\tau_{1 / 2}$ is the time for the potential to drop to the value of $\gamma$ (in this case, $40 \mathrm{~cm}^{-1}$ ). Accordingly, the bond is broken at $4 \AA$. As expected, this value for the length parameter is smaller than the previously discussed, unrealistic value of $5.3 \AA$, which assumed the terminal velocity throughout the fragment motion.

By increasing the pump energy, and so reducing the initial internuclear separation $R_{0}$, we can test the effect of recoil velocity and available energy. In this case, the pump wavelength was reduced from 306 to $285 \mathrm{~nm}$. Two competing effects are involved: On the one hand, the distance from $R_{0}$ to the probe OCR becomes greater, which would tend to increase $\tau_{1 / 2}$. On the other hand, as we increase $E$, the recoil velocity increases and $\tau_{1 / 2}$ will shorten. Since the rotational internal energy of the fragments is known $\left(\sim 2000 \mathrm{~cm}^{-1}\right.$ at $285 \mathrm{~nm}$ vs $\sim 1200 \mathrm{~cm}^{-1}$ at $308 \mathrm{~nm}$ ), ${ }^{12}$ the available (and translational) energy is increased, so that the final recoil velocity is larger. For ICN, at $285 \mathrm{~nm}$, the $\mathrm{I}^{*}$ channel is energetically possible, and there exists the possibility of an avoided crossing between the two channels that lead to I and $I^{*}$. Such a crossing, if it occurred, might substantially affect the dynamics of the reaction, leading, for example, to long delays or to oscillations (resonances) like those observed for the alkali halides. ${ }^{30}$

Our results indicate that tuning the pump to $285 \mathrm{~nm}$ is associated with a net reduction in $\tau_{1 / 2}$, to $160 \pm 30 \mathrm{fs}$. This suggests that the predominant effect involved is due to the increased velocity of fragment recoil. Considering Eq. (4), $\tau_{1 / 2}$ at the two available energies are consistent with the same length parameter of the potential. No direct evidence has yet been obtained that the crossing from the $I^{*}$ channel to the $I$ channel delays the appearance of free- $\mathrm{CN}$ or leads to resonances. This is consistent with the idea that such crossings are at shorter $\boldsymbol{R}_{0}$, near the Franck-Condon region. However, work is continuing in this area, and the possibility exists that these phenomenon may be observed if the pump wavelength is tuned to wavelengths shorter than $285 \mathrm{~nm}$ or if the pulse widths are further shortened.

The effect of the spectral width of the pulses $(\gamma)$ was studied in two ways: (1) by changing $\Delta v$ and $\Delta t$ of the pulses over a limited range in a way that maintained transformlimited pulses (accomplished by adjustment of the CPM laser), and ( 2 ) by spectrally broadening the pulses (by slight saturation in the PDA), which produced pulses with $\Delta v$ $\Delta t \geqslant 1$. Even though the effect of $\gamma$ in Eq. (4) is relatively insensitive, we observed a consistent trend for $\tau_{1 / 2}$ vs $\gamma$ for the many experiments we did. These results were also consistent with previous measurements ${ }^{14}$ made with subpicosecond pulses, where the full rise was found to be in $\sim 600 \mathrm{fs}$. We have noted, however, that the existence of a chirp in the optical pulses could affect the timing of an on-resonance rise, because the exact $t=0$ may not be well defined when each wavelength component of the pump arrives at a slightly different time. This would not seem to be the predominant effect in our nontransform limited data, since we have repeated the experiments for probe spectra centered at slightly different wavelengths and these obtained values of $\tau_{1 / 2}$ were self-consistent.

It is interesting to note the similarity in shape of the two traces in Fig. 4. As detailed in paper I, the FTS transient should approximately equal the response function (which is the integral of the cross correlation) in the limit where the intrinsic spreading of the wave packet on $V_{1}$ is ignored or, 
more exactly, if the spreading is on a shorter time scale than the duration of the pump and probe pulses. Within the experimental errors, the two traces are not significantly different, so that at this time we cannot address with confidence the question of packet dispersion.

In conclusion, these clocking experiments provide an operational way to obtain a characteristic femtosecond timeof-flight $\tau_{1 / 2}$ for a chemical bond. This $\tau_{1 / 2}$ can be related to the dynamics of bond breaking and the "window" (the OCR) opened on the potential by the probe.

\section{B. Detuning experiments: To the red}

For ICN, tuning the probe wavelength to the red of the bandhead at $\lambda_{2}^{\infty}=388.5 \mathrm{~nm}$ leads to the observation of femtosecond-lived transition states. ${ }^{2}$ In Fig. 5, the results for FTS experiments for several values of $\lambda_{2}=\lambda_{2}^{*}$ [i.e., $A(t$; $\left.\left.\lambda_{2(\mathrm{a})}^{*}, \lambda_{2(\mathrm{~b})}^{*}, \ldots, \lambda_{2}^{\infty}\right)\right]$ are presented. The on-resonant case, $\lambda_{2}=\lambda_{2}^{\infty}$, was discussed in the last section. The FTS transient behavior characteristically was a simple rise (after a delay $\tau_{1 / 2}$ ) to a limiting value, which represented the buildup of population into the final state. The other transients in Fig. 5 shows some representative results obtained for offresonant probe wavelengths, $\lambda_{2}=\lambda_{2}^{*}$. In the detuned experiments, the behavior is seen to be a rise followed by a decay to some asymptotic level. The height of the asymptote is a function of the probe wavelength. As predicted in paper
I, this level decreases as $\lambda_{2}$ is tuned away from $\lambda_{2}^{\infty}$. Note that for $\lambda_{2}^{*}=391.4 \mathrm{~nm}$ in Fig. 5, the decay of the transient is virtually complete. Such behavior has been repeatedly observed in many experiments not shown here (we only present these four typical sets), and is consistent with the measured probe spectrum as discussed below.

The decay in some cases is on a longer time scale than the pulses, but in other cases is within our time resolution, depending on $\lambda z_{2}^{*}$. We use the experimentally measured cross correlation to correct for this convolution effect. To illustrate the effect of pulse convolution on our transients, we present in Fig. 6 some simulations.

We now apply the simple theories discussed in paper I to these detuned experiments. The kinetic model in Sec. V A of I describes the height of the asymptote by the inclusion of a tuning parameter $\beta$. Tuning of the probe as in Fig. 5 would correspond to changing $\beta$ from 0 (completely on-resonant case) to 1 (completely off-resonant case). The data presented in Fig. 5 can be well fit by the six-level approach (see Fig. 11 of paper I). The spontaneous lifetimes obtained from the parent to the transition state and from there to the final product state $\left(1 / k_{b c}\right.$ and $1 / k_{c e}$, respectively $)$, are of the expected order-of-magnitude, $\sim 10^{-13} \mathrm{~s}$. As pointed out in $\mathrm{I}$, the use of the kinetic approach is heuristic because the continuum of transition states is replaced by a single, discrete level.

A more meaningful description is obtained using the
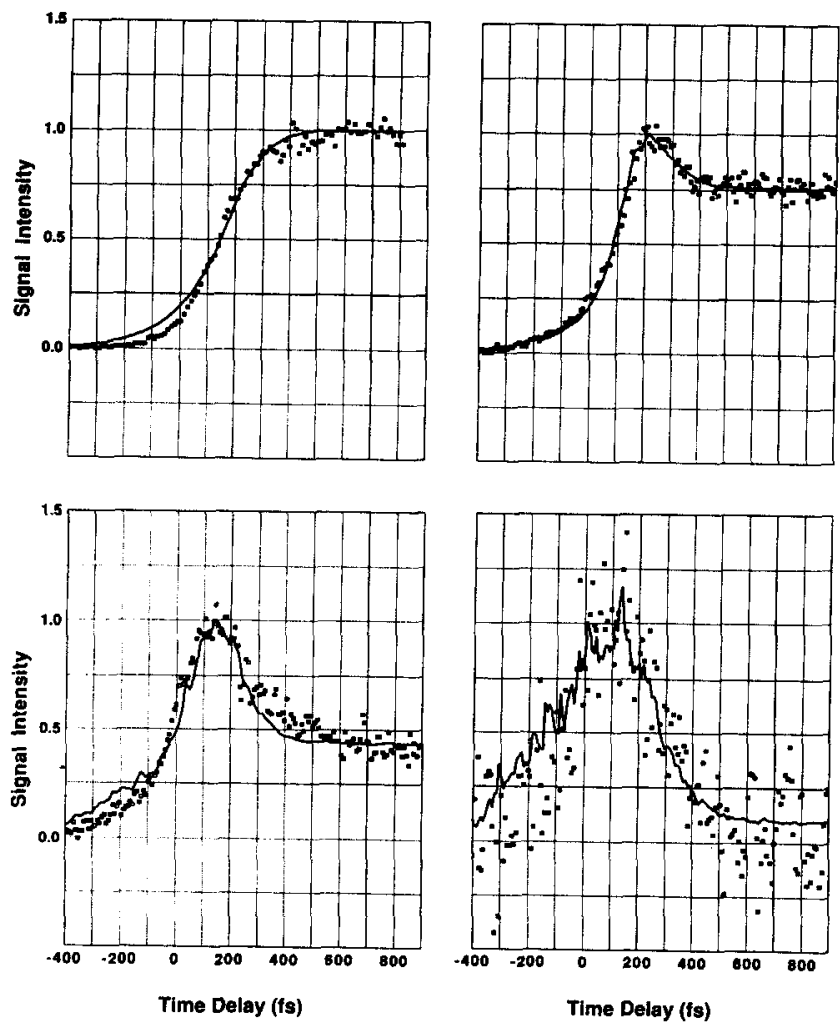

FIG. 5. Typical ICN transients as a function of probe wavelength. The probe wavelengths were (a) $\lambda_{2}^{*}=388.9 \mathrm{~nm}$, (b) $\lambda_{2}^{*}=389.8 \mathrm{~nm}$, (c) $\lambda_{2}^{*}=390.4 \mathrm{~nm}$, and (d) $\lambda_{2}^{*}=391.4 \mathrm{~nm}$. The solid lines were generated by fitting the classical model of Eq. (5) convolved with the measured experimental response for each transient.
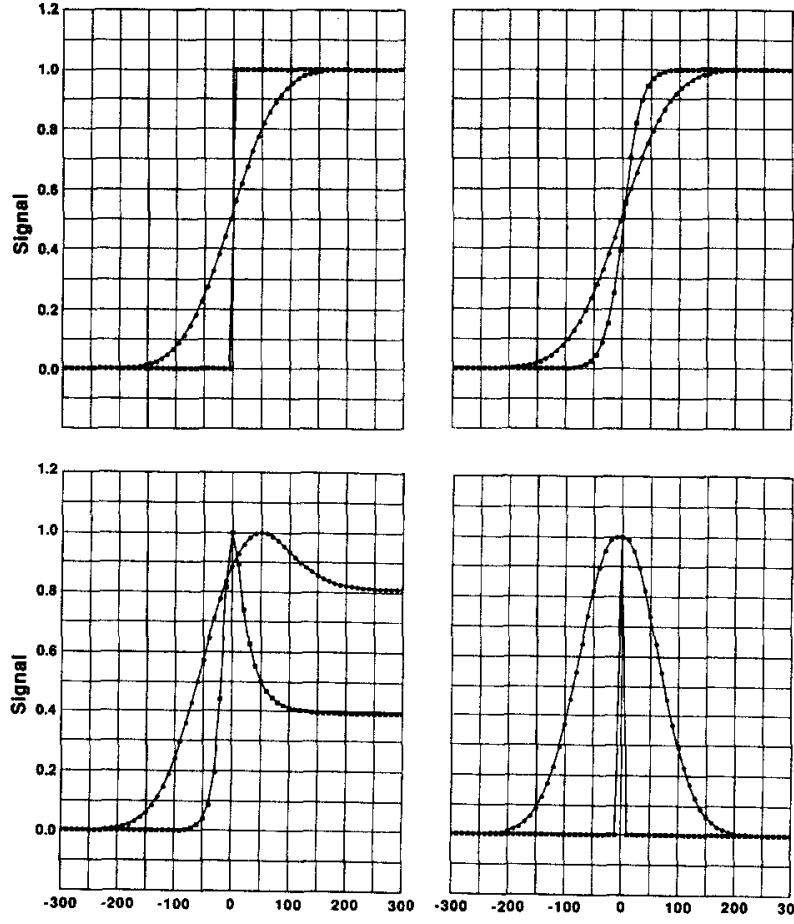

Time Delay (fs)

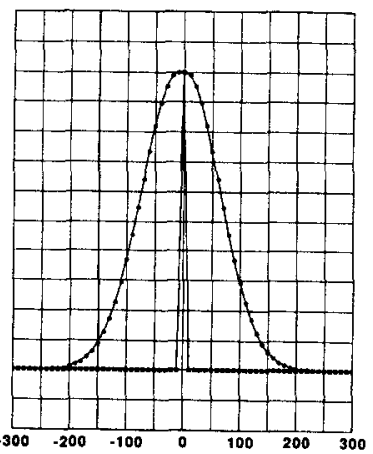

TIme Delay (fs)

FIG. 6. Simulation of the effect of convolution on the transients. The numerical results (solid circles) are the convolutions of a Gaussian pulse of 150 fs width with the following assumed shapes (open squares): (a) an ideal step function; (b) Eq. (5) of the text, with $t^{*}=\infty$ (on-resonance); (c) Eq. (5) of the text, with $t^{*}<\infty$ (off-resonance); and (d) a delta function. Note that $\tau_{1 / 2}$ is not affected by the convolution in cases (a) and (b). 
classical mechanical model ${ }^{29}$ presented in I. Here, we shall invoke two assumptions which will be discussed below: the excited state PES $\left(V_{1}\right)$ has an exponential shape, and the upper state potential satisfies a condition to be discussed shortly. The absorption of the probe photon as a function of time is then given by Eq. (20) of paper I:

$$
A(t)=\frac{C}{\gamma^{2}+W^{2}\left(t, t^{*}\right)},
$$

where

$$
W\left(t, t^{*}\right)=E\left\{\operatorname{sech}^{2}\left[\frac{v t}{2 L_{1}}\right]-\operatorname{sech}^{2}\left[\frac{v t^{*}}{2 L_{1}}\right]\right\} .
$$

In this expression, $t^{*}$ is defined to be the time at which the absorption achieves a maximum, $C$ is a constant, and $L_{1}$ is the distance scale of $V_{1}$.

The solid lines in Fig. 5 are the best fit results obtained by the convolution of Eq. (5) with the experimentally measured cross correlations. The underlying (deconvolved) absorptions are shown for each of these fits in Fig. 7. The only parameters allowed to vary in these fits are $t^{*}$ and $L_{1}(\gamma$ is determined by the probe spectrum), and the results are presented in the caption.

These fits raise a number of interesting issues. First, there is good consistency among all the best-fit values for $L_{1}$ ( $\approx 0.8 \AA$ ) in providing $t *$ values that are systematically ex-
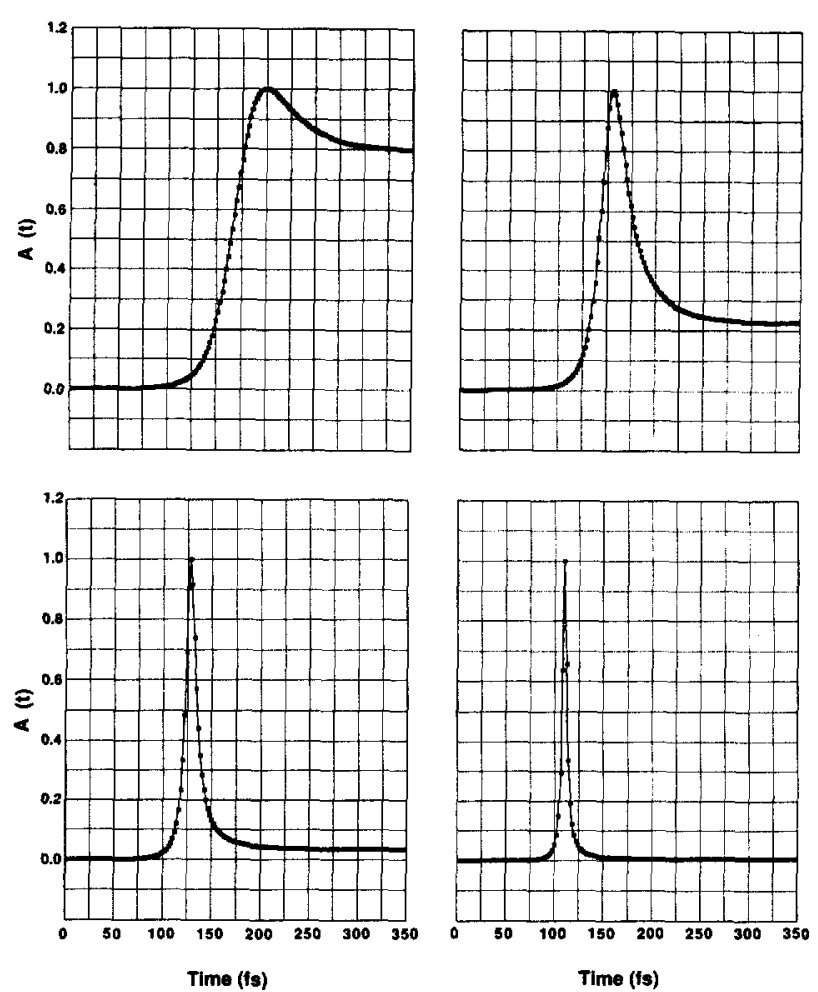

FIG. 7. Deconvolved fits to the detuned experiment results. The fits presented in Fig. 5 are shown with the experimental response deconvolved. Fit values are: (a) $L=0.84 \AA, t^{*}=200 \mathrm{fs}, \gamma=96.5 \mathrm{~cm}^{-1}$; (b) $L=0.84 \AA$, $t^{*}=156 \mathrm{fs}, \gamma=102 \mathrm{~cm}^{-1}$; (c) $L=0.84 \AA, t^{*}=128 \mathrm{fs}, \gamma=83.5 \mathrm{~cm}^{-1}$; and (d) $L=0.88 \AA, t^{*}=110 \mathrm{fs}, \gamma=64 \mathrm{~cm}^{-1}$. pected to occur at shorter times. In addition, this value is in agreement with that obtained for $L_{1}$ using the clocking approach, $t^{*} \rightarrow \infty$ (see last section). The closeness of these fits to the measured data implies that

$$
\begin{aligned}
& V_{2}^{0} \exp \left\{-\left[R\left(\tau_{1 / 2}\right)-R_{0}\right] / L_{2}\right\} \\
& \quad \ll V_{1}^{0} \exp \left\{-\left[R\left(\tau_{1 / 2}\right)-R_{0}\right] / L_{1}\right\} .
\end{aligned}
$$

This condition is satisfied either by making $L_{2}$ less than $L_{1}$, or else by $V_{2}^{0} \ll V_{1}^{0}$, and is consistent with our observation of a red shift. We have made simulations of the more general case to test the effect of $V_{2}^{0}$ and $L_{2}$ and found consistency in the derived value of $L_{1}$. (We will detail this study later.) The potential surfaces of ICN cannot be expected to be exactly exponential in shape throughout, because of the existence of long-range interactions and van der Waals wells. However, the exponential shape discussed gives an idea of the distance scale involved. The value of $L_{1}$ obtained here is about a factor of 4 larger than the value $(\sim 0.2 \AA)$ obtained by several authors ${ }^{31}$ from the absorption spectrum continuum in the Franck-Condon region. This is very reasonable in light of the fact that, in the Franck-Condon region (at short $R$ 's), the potential is very steep, and in the long-range part we expect a different slope (less steep). The inner shell repulsions usually have a much shorter range than the attractive forces, which might cause the net repulsion to change its shape at the longer distances.

We now consider the variation with respect to $t *$ in this data. Note that in Figs. 5 and 7, the time delay is given in absolute units. Determination of the $t=0$ timing was made for each data set using the multiphoton ionization technique previously described. The time delay at which the FTS signal achieves its maximum moves towards earlier times as the detuning is increased. The systematic variation in the peak position is even more apparent in the deconvolved results, Fig. 7. This is a crucial observation, required by the models of FTS discussed in paper I. Because the transition induced by the probe from $V_{1}$ to $V_{2}$ is vertical, the choice of probe wavelength determines the OCR on the PES. For instance, if $\lambda_{2}=\lambda_{2}^{\infty}$, then the OCR will extend from some finite internuclear separation (whose value depends on the spectral bandwidth of the probe) to infinity. As the probe wavelength is increased, the OCR moves towards smaller intermuclear separation for the case under consideration (red shift). Since the FTS experiment is a clock of the time required to move from $R_{0}$ to the OCR, the transient must therefore reach its maximum at successively shorter times as the wavelength is tuned further to the red. ${ }^{32}$

This last point is important, as it shows that the detuning experiments can be interpreted as a generalization of the clocking technique. For each probe wavelength, the value of $t^{*}$ has been determined. If the location of the probe OCR [i.e., $R\left(t^{*}\right)$ ] is known for that pulse, then the average velocity required to reach there is deduced. The more general situation, in which the PES is determined without such knowledge, is discussed below.

The transition state $[\mathrm{I} \cdots \mathrm{CN}]^{\ddagger *}$, persists only for a finite time, and its duration is measured in this detuning experiment. In Fig. 7, for example, the deconvolved absorption persists only for $\sim 20-50$ fs. According to the model, the 
time required to traverse the full width of the absorption at half-maximum is

$$
\tau^{*} \equiv 2\left(t-t^{*}\right) \approx \sqrt{2} f L_{1}^{*} / v^{*},
$$

where $f$ is in this case $\sim 1$, and $v^{*}$ is the speed of separation at $R=R *$. The length factor $L_{1}^{*}$ (the length in which the potential changes by $\gamma$ at the transition-state probing) is simply $\gamma L_{1} / V_{1}\left(R^{*}\right)$. The quantity $V_{1}\left(R^{*}\right) / L_{1}$ is the rate of change of $V_{1}(R)$ with distance at $R=R *$. To obtain transition-state "lifetimes" of the order of $50 \mathrm{fs}$, this suggests that the potential dropped to a hundred wave numbers or so, as shown below.

\section{Tuning to the blue}

The LIF spectrum of free-CN consists not of a single transition at $388.5 \mathrm{~nm}$, but exhibits the many transitions of the $P$ and $R$ branches. For $308 \mathrm{~nm}$ excitation, the $R$ branch is fully resolved and extends to $381.5 \mathrm{~nm}$, as shown by Fisher $e t$ $a l .^{34}$ (In addition to $v=0$, the ground-state vibrational level, rotational lines, much weaker lines due to $v=1$ were also reported.) These transitions to the blue of the bandhead are associated with different rotational quantum numbers of the $\mathrm{CN}$ fragment (up to $\sim 50$ ), and as mentioned in the Introduction have been analyzed to give product state distributions.

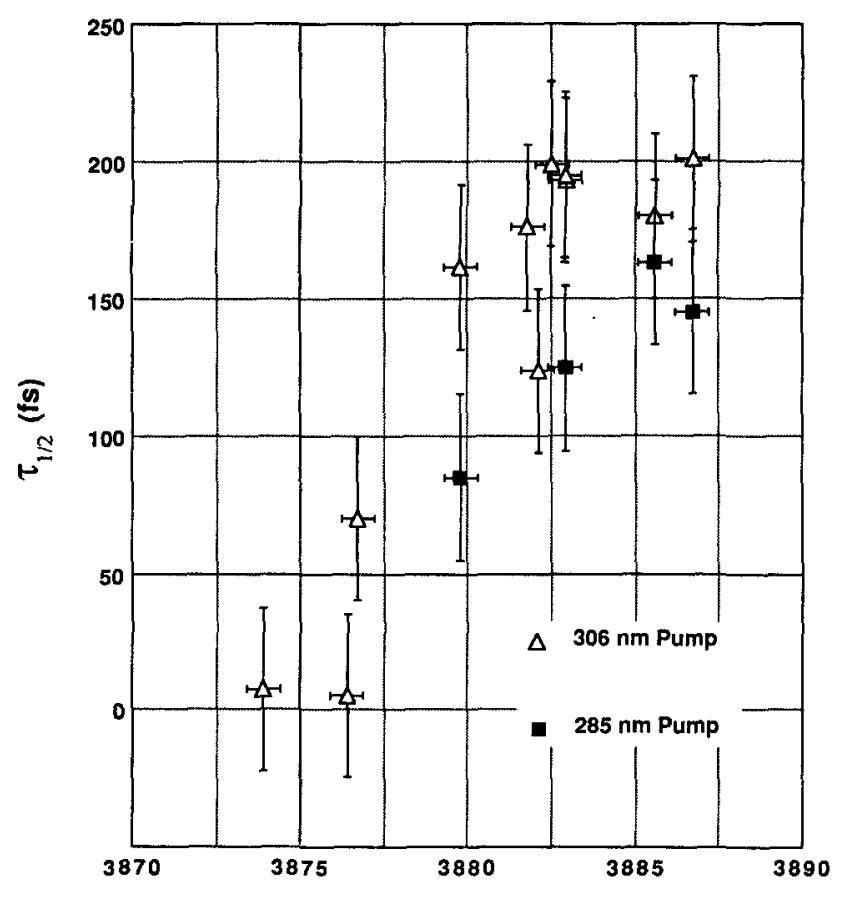

Wavelength (A)

FIG. 8. Delay time as a function of probe wavelength. The values of $\tau_{1 / 2}$ obtained are shown for an approximate pump wavelength of $306 \mathrm{~nm}$ (open triangles) and $285 \mathrm{~nm}$ (solid squares). The FWHM of the probe was $\sim 60$ $\mathrm{cm}^{-1}$ in each case.
The spectral width of the femtosecond probe is broad, so that the excitation of a single rotational level is impossible. However, we have attempted to selectively probe regions within the $P$ branch of the $C N$ spectrum which are associated with either high or low rotational quantum states. For example, the region near the bandhead is associated with high rotational states $\left(N^{\prime \prime} \geqslant 20\right)$, and near the start of the $P$ branch at $387.5 \mathrm{~nm}$, with lower quantum numbers.

The clocking experiment was performed at various probe wavelengths between 388.7 and $387.4 \mathrm{~nm}$. The values of $\tau_{1 / 2}$ determined from these measurements are shown in Fig. 8, for both 306 and $285 \mathrm{~nm}$ pump wavelengths. For pulses at the bandhead, as previously discussed, this experiment yields a time delay of $\tau_{1 / 2} \sim 200 \mathrm{fs}$. However, a substantial decrease in $\tau_{1 / 2}$ was observed as the probe was shifted towards shorter wavelengths. ${ }^{35}$

An obvious possible cause for this observation is due to the change in the translational energy with rotational excitation of the CN. This is illustrated in Fig. 9 . For $7000 \mathrm{~cm}^{-1}$ of total available energy, the low $N^{\prime \prime}$ states will be produced with essentially all this energy in translation. However, for high $N^{\prime \prime}$ states the translational energy will be less than the available energy; for $N^{\prime \prime}=25, E_{R}=1250 \mathrm{~cm}^{-1}$, and the translational energy becomes $5750 \mathrm{~cm}^{-1}$ (vibrational excitation is assumed to be negligible ${ }^{34}$ ). In the center-of-mass frame, $v$ is proportional to $E^{1 / 2}$, and $\tau_{1 / 2}$ at most should change from $\sim 200$ to $\sim 180$ fs. This is a much smaller effect than the data in Fig. 8 shows.

Probing the different $N^{\prime \prime}$ states of the $\mathrm{CN}$ is sensitive to a more interesting dependence, namely, the angular part of the potential. In general, the PES is not only radial but it has an angular dependence described by the angle $(\theta)$ between I and the center-of-mass of the $\mathrm{CN}$. For ICN, the potential has been postulated and used to fit the product state distribution, e.g., as done by Goldfield et al. ${ }^{12}$ A form that is discussed by Schinke ${ }^{36}$ and others is generally written as

$V(R, \theta)=A \exp \left[-\frac{R-R_{0}}{L}\right] \exp \left[-\frac{\epsilon\left(\cos ^{2} \theta-1\right)}{L}\right]$,

where $\epsilon$ is a measure of the coupling between the dissociation coordinate $R$ and $\theta$. When $\theta=0$, we recover the single radial potential (isotropic). But for negative $\epsilon$, we have a bent ex-

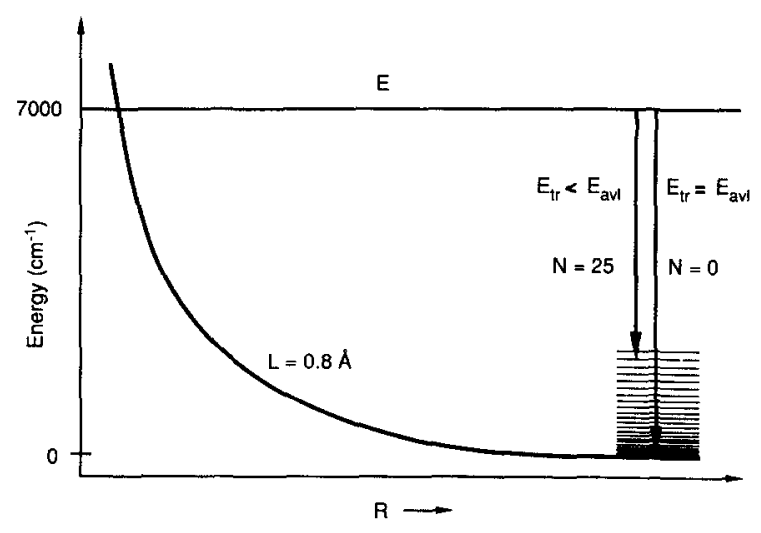

FIG. 9. Change in translational energy with rotational energy of $\mathrm{CN}$. The probe pulse either samples regions of high or low rotational quantum numbers. 
cited state which has a minimum at $\theta=90^{\circ}$ and a maximum at $\theta=0$. Dugan ${ }^{16}$ has discussed this bending ( of $\mathrm{CN}$ relative to 1 ) at the $308 \mathrm{~nm}$ excitation and obtained a bending angle of $\theta_{0}=18^{\circ}$. Basically, if the molecule were linear $\left(\theta_{0}=0\right.$, on the average), then the rotational distribution would show a maximum at $N^{\prime \prime} \approx 0$; instead, the maximum seen in these experiments is at higher $N^{\prime \prime}$ values for this wavelength of excitation. As he pointed out, at these long wavelengths there is one or two quanta of bending vibration (ground state), and their classical turning points are at $14^{\circ}$ and $19^{\circ}$. We will not attempt quantification of the angle here, but the important points are that the bent state produces a range of torques and that there is time evolution for $N$.

Elsewhere, ${ }^{37}$ we will show that such bending may describe the observation. At shorter times the rotational distribution is biased toward lower $N$ states, while at longer times all states are produced. Because of bending and the centrifugal potential, probing higher $N$ states of the distribution will cause a longer delay than the lower $N$ states. Such changes should be directly related to $V(R, \theta)$ and simulations are underway to quantify this effect and consider other channels. It should be noted that the clock used here to measure $\tau_{1 / 2}$ is sensitive to the separation of the I and CN (and any changes in the $\mathrm{CN}$ bond length). Even though the bending may develop at earlier times compared to the recoil times, it has a signature on the different angular momentum states of $\mathrm{CN}$, and the total time it will take a trajectory to make it to a given $N$ state will depend on $V(R, \theta)$.

\section{Femtosecond coherence and alignment}

For these experiments, we used a polarized femtosecond pulse arrangement to probe the degree of coherence and alignment as a function of time. Figures $10(a)$ and $10(b)$ each show two data sets, taken under identical conditions, one with parallel, and the other with perpendicular polarizations of the pump and probe beams. The data has been normalized to an asymptotic value of one, and (for the lower data set only) the absolute zero-of-time has been determined. The data on the top set is for a bluer wavelength of probing, and was done under entirely different conditions.

The characteristic results of these femtosecond alignment experiments are as follows: (1) the parallel polarization transient achieves a larger normalized amplitude in the first hundred femtoseconds or so after pump excitation, (2) the general shape of the transient is similar, and (3) the time delays at which the signals achieve their maximum values are equal ( to within experimental resolution ). The change in amplitude for $A_{\|}(t)$ and $A_{1}(t)$ is not very large but reproducible in these two different sets of experimental results. It is possible that when we complete these measurements using shorter pulses that we will be able to resolve the anisotropy $A_{\|}(t)-A_{1}(t)$ better. $^{38}$

As the $\mathrm{CN}$ separates from the $\mathrm{I}$ atom, the $\mathrm{CN}$ rotational states evolve in a "coherent packet" which then dephases to an incoherent product state population. The time evolution for this process determines the degree of alignment $\left[A_{\|}(t)\right.$ and $\left.A_{1}(t)\right]$ which depend on $P_{2}\left(N \omega_{0} t\right)$, where $P_{2}$ is the second Legendre polynomial. If the $\mathrm{CN}$ is produced in, for in-
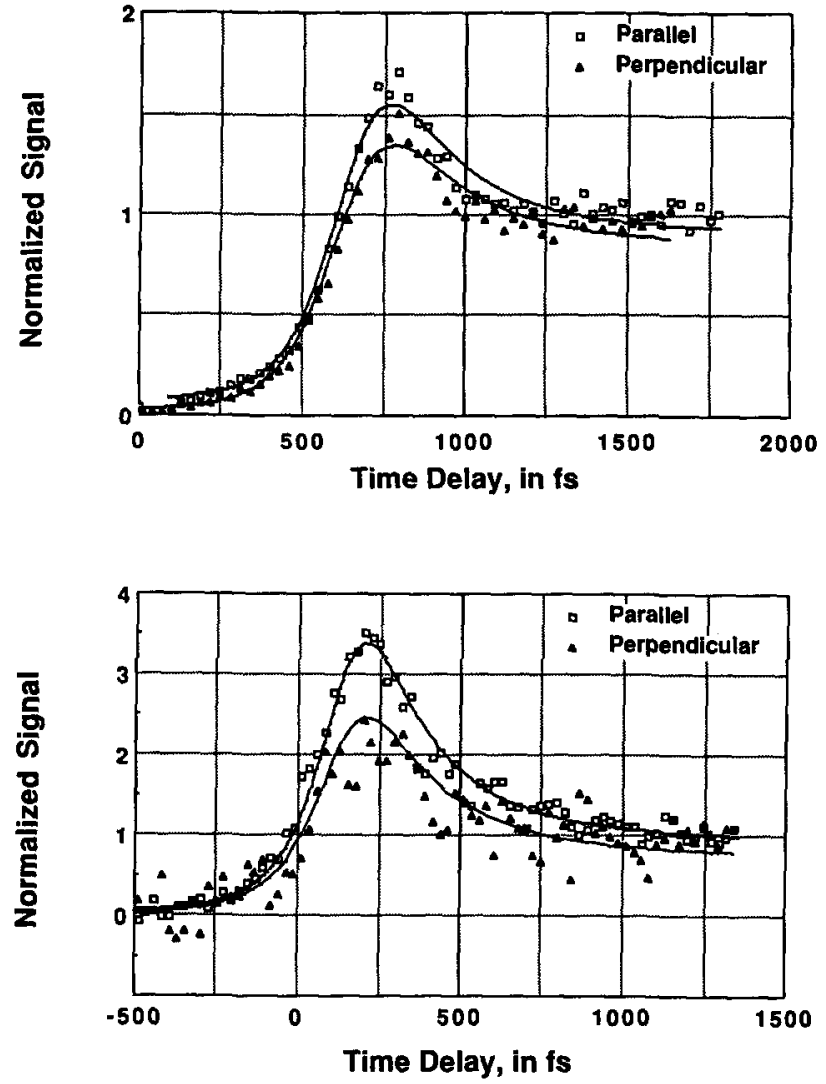

FIG. 10. Results of femtosecond alignment experiments. FTS data taken off-resonance, with either parallel (open squares) or perpendicularly polarized (solid triangles) pump and probe beams. The probe polarization was kept parallel to the detection axis. Two data sets are shown, taken under different experimental conditions. Note that the time delay in the upper curve is arbitrary (the zero-of-time was not determined). The data is normalized to a value of unity at long time delays. The lines are guides to the eye.

stance, a few very low $N$ states, then the coherence time will be on the order of the inverse of the rotational constant; it becomes shorter for larger $N$. In this case, beats (with inphase and out-of-phase characteristics) for the two polarizations will be observed. On the other hand, if the $\mathrm{CN}$ is produced in a distribution of $N$ states, then dephasing of the initial coherence will result in an initial decay and some recurrences. ${ }^{24}$ For the problem at hand, taking the dissociation as an impulse to excite the $\mathrm{CN}$ coherently with a distribution of rotational energies, the coherence time in the course of dissociation can be estimated by ${ }^{37}$

$$
\tau_{c} \approx \frac{2.2}{\sqrt{B\left(E_{R}\right\rangle}},
$$

where $\tau_{c}$ is in picoseconds and both the constant $B$ and the average rotational energy $\left\langle E_{R}\right\rangle$ are in $\mathrm{cm}^{-1}$. At our excitation wavelength, this gives $\tau_{c} \approx 44 \mathrm{fs}$.

As discussed elsewhere, ${ }^{37}$ one can write expressions for $A_{\|}(t)$ and $A_{1}(t)$ which include explicitly $\tau_{c}$, and show the relative importance of $\tau_{c}$ to $\tau^{*}$ and $t^{*}$. If the coherence time is long compared to $t^{*}$, then both $A_{\|}(t)$ and $A_{1}(t)$ should have similar shapes and will only differ by a constant scale 
factor of 3. However, if the coherence time is sufficiently short relative to our pulse, the initial part of the transient will be convolved. The similarity of the transient is consistent with this picture, and our observations, therefore, suggest that the coherence time is on the time scale of our experiment. Shorter pulses will help resolve this effect and obtain accurate $T_{c}$. The shorter the $t^{*}$ of observation, the larger the anistropy should be, consistent with the preliminary data in Fig. 10. (Note the values of the asymptotes and the initial anisotropy.) Wilson's group is proposing this type of anisotropy experiments to probe changes in the dynamics of the potential ongoing from the gas phase to the condensed phase. ${ }^{39}$

\section{E. The potential energy surface}

From knowledge of the length parameter $L$ obtained above, we can now deduce the change of the potential with time $V(t)$ and the corresponding $V(R)$. We will use the same approximation invoked in Sec. III B. The experimentally measured $A(t)$ can be used to obtain the explicit form of $V_{1}(t)$ :

$$
V_{1}(t)=V_{1}\left(t^{*}\right) \pm \gamma \sqrt{\frac{A_{\max }}{A(t)}-1},
$$

where $A_{\max }$ is a constant (given by $C / \gamma^{2}$ in I). The $+(-$ ) sign is used for $t<(>) t^{*}$. For an exponential potential in $R$, this $V(t)$ will be explicitly given by

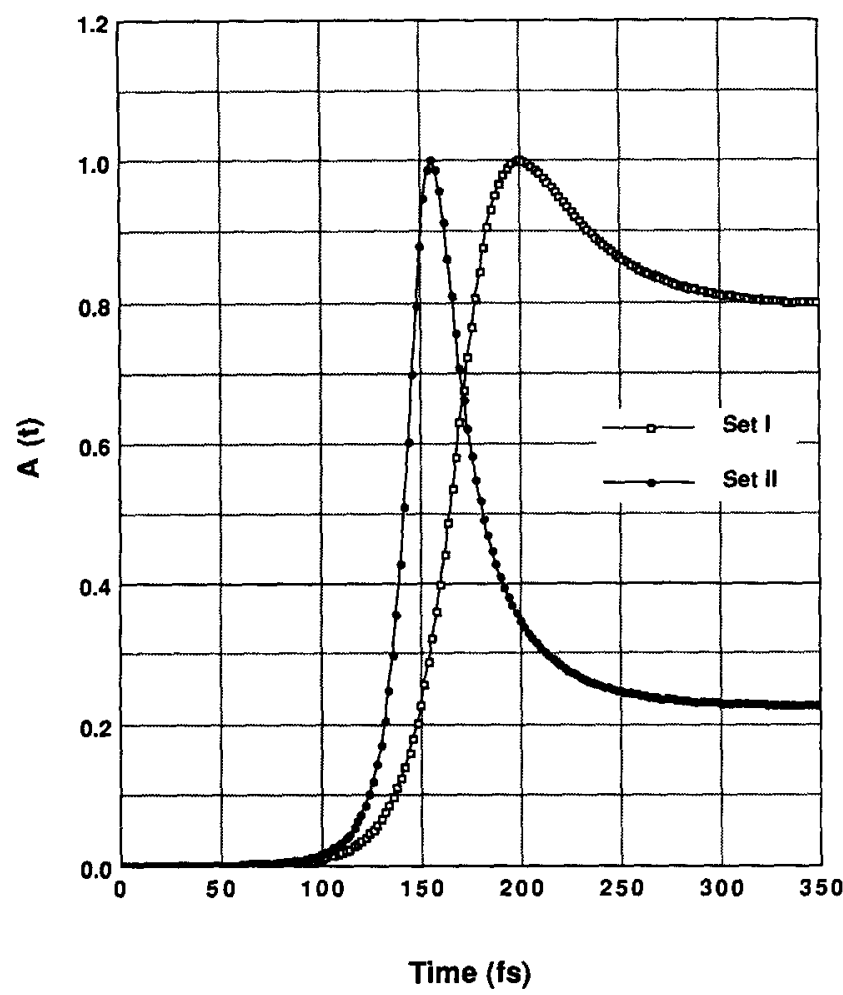

FIG. 11. Deconvolved absorption from detuning experiment. Data taken from Figs. 7(a) (open squares) and 7(b) (solid circles). Note the time shift between the two transients. The two transients will be used for the inversion shown in Fig. 12.

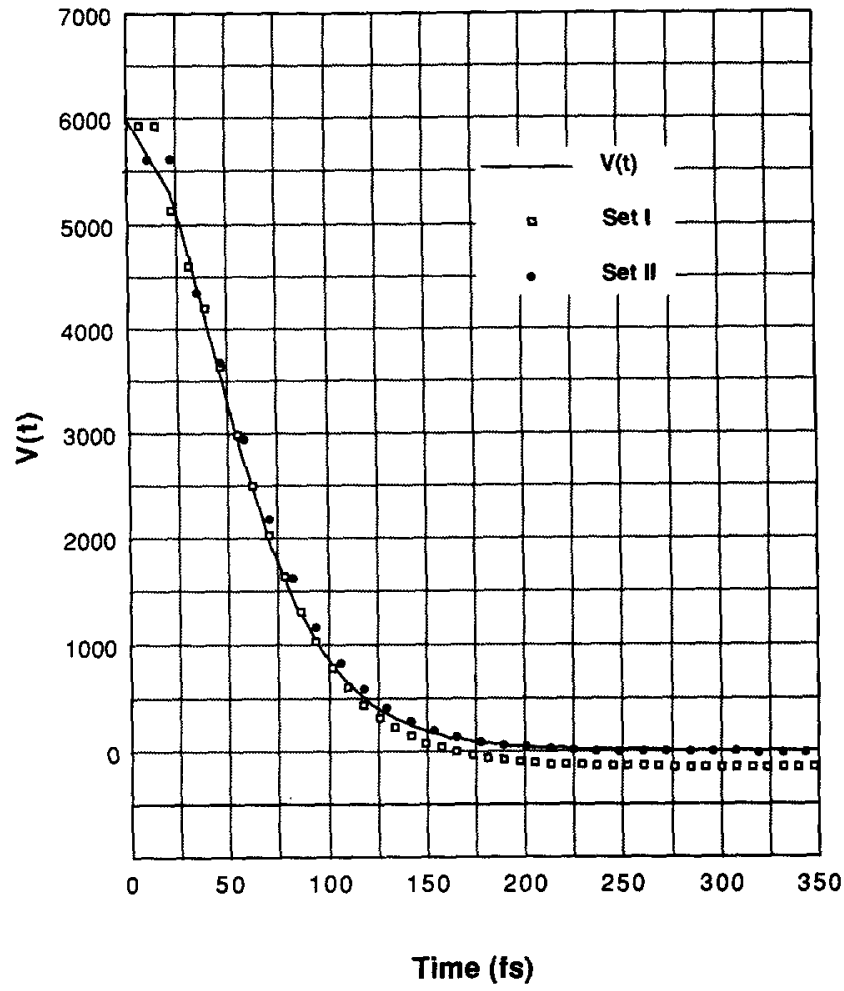

FIG. 12. Inversion to the potential. The calculation of the potential as a function of time, $V(t)$, is shown for the data of Fig. 11. The solid line is the theoretical result [Eq. (10)] using the fit value for $L_{1}$.

$$
V_{1}(t)=E \operatorname{sech}^{2}\left(v t / 2 L_{1}\right) .
$$

Figure 11 shows two (deconvolved) sets of experimental transients, $A(t)$ vs $t$. Using Eq. (9), we have inverted to obtain $V_{1}(t)$ (Fig. 12), and as expected we obtained the presumed potential, which has dropped down to a negligible value in $\sim 200 \mathrm{fs}$. It is important to note that the shape of $V_{1}(t)$ vs $t$, especially at short times, is not exponential (see also Fig. 13). This is because the recoil velocity changes with

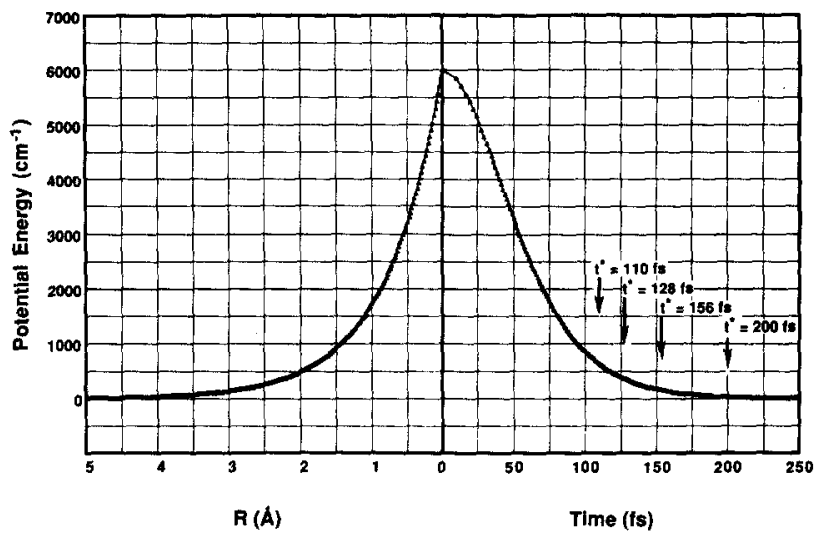

FIG. 13. The potential as a function of time and length. $V$ is shown as a function of the internuclear separation (left) and time (right). The values at left show an exponential shape with the experimentally deduced length parameter, and those at right are derived from Eq. (10). The values of $t^{*}$ from the fits of the data of Fig. 5 are indicated. 
time; at early times the fragments separate slowly, but eventually accelerate to the terminal velocity. For this same potential we display in Fig. 13 the corresponding $V_{1}(R)$ and $t^{*}$ for four sets of probes. This helps illustrate the corresponding distance scales involved.

One drawback of the above procedure is the assumption made about the upper surface. Naturally, we wish to invert to the potential without any $a$ priori assumptions about the shape of $V_{2}$ (exponential repulsion, $R^{2}$, etc.). There is a method by Bernstein and Zewail ${ }^{33}$ which inverts to give $\Delta V(t)$ vs $t$ (i.e., the change in the difference potential with time) and, by pump-tuning experiments, $V_{1}(t)$. This method will be described in Ref. 33 and we briefly mention it here to make a connection with the above discussion. The idea is as follows: For a given $t^{*}, A(t)$ is known as a function of $t$. Also, $A(\Delta)$ is known, where $\Delta$ is the detuning from $\lambda_{2}^{\infty}$ and $A(\Delta)$ is a measure of the amount of absorption of the free fragment as a result of the finite width of the pulse. Therefore, by projection of $A(\Delta)$ vs $\Delta$ onto $A(t)$ vs $t$, we obtain $\Delta V$ vs $t$ without $a$ priori assumptions on the shape. In the future, we expect two improvements in order to construct the PES over a larger range of $R$ : the detuning should be extended, to values of $\lambda_{2}^{*}$ further to the red (and blue), and the pulse widths should be narrowed. Such experiments are feasible.

A final note should be made about the quantum mechanical simulation of the ICN reaction. As mentioned in paper I, Williams and Imre ${ }^{40}$ have simulated the wave packet for dissociation of ICN (see Fig. 16 of I) as a function of detuning. Their results are in good agreement with the experimental shape of the transients (see Fig. 17 in I). In their simulation, they used $\alpha=1 / L_{1}=(0.33 \AA)^{-1}$, and they simulate the different experiments at different $\lambda_{2}^{*}$ (388.9, 389.5 , and $390.5 \mathrm{~nm}$ ). Note the consistency in the time scale, time shift, and the magnitude of the asymptotes with the experiments. When $\alpha$ is rescaled to agree with our experimentally deduced value of $(0.8 \AA)^{-1}$, the expected time delay (or wave packet displacement to dissociation) is close to our $\tau_{1 / 2}$. It is pleasing to see that the quantum wave packet model is reproducing the experimental features very well, and is in agreement with the classical predictions. There are, of course, many details that have not been quantified yet. We are currently examining details of the PES and their influence on the shape and the movement of the packet, under different experimental conditions of interest.

\section{CONCLUSIONS}

In this study, we have applied FTS to the dissociation reaction of ICN. The breaking of the I-CN bond has been clocked and the characteristic time $\tau_{1 / 2}$ has been measured. Short-lived transition states of the reaction $\left([\mathrm{I} \cdots \mathrm{CN}]^{\ddagger *}\right)$ were observed in experiments where the probe pulses were detuned to longer wavelength than the bandhead of the free$\mathrm{CN}$ transition. Both the on-resonance (clocking) and offresonant (buildup and decay) behaviors are reproduced theoretically using classical and quantum mechanical models of FTS presented previously in paper I. From the experimental observations and using an exponential repulsion, the length scale for the PES of ICN was deduced for the range of $R$ studied. Tuning the probe frequency to the blue yielded smaller values of $\tau_{1 / 2}$, which we relate to the angular part of the PES, and can be explained by the consideration of the femtosecond time evolution of the different rotational quantum numbers. Femtosecond alignment studies of ICN, as well as theory, suggest that coherence during dissociation is lost rapidly, estimated to be $\sim 44 \mathrm{fs}$.

This paper gives a methodology for treating these first FTS studies of reactions, and work is continuing on ICN and other reactions to invert the FTS data and obtain more accurate PES. We limited ourselves here to simple descriptions, but there are a number of theoretical and experimental issues to be further developed. As we gain more experience in this field, we hope to exploit the full capabilities of these FTS techniques and learn more about real-time femtosecond dynamics (population and alignment) of elementary reactions.

\section{ACKNOWLEDGMENTS}

This work was supported by AFOSR (Grant No. 870071). Contribution No. 7814. We wish to thank the referee of this and the previous paper for a thorough and comprehensive review.

'M. J. Rosker, M. Dantus, and A. H. Zewail, J. Chem. Phys. 89, 6113 (1988).

${ }^{2}$ M. Dantus, M. J. Rosker, and A. H. Zewail, J. Chem. Phys. 87, 2395 (1987).

${ }^{3}$ G. W. King and A. W. Richardson, J. Mol. Spectrosc. 21, 339 (1966); see also Ref. 8(b).

${ }^{4} \mathrm{H}$. Okabe, Photochemistry of Small Molecules (Wiley-Interscience, New York, 1978).

${ }^{5}$ A. P. Baronavski and J. R. McDonald, Chem. Phys. Lett. 45, 172 (1977).

'J. H. Ling and K. R. Wilson, J. Chem. Phys. 63, 101 (1975).

${ }^{7}$ (a) W. Krieger, J. Hager, and J. Pfab, Chem. Phys. Lett. 85, 69 (1982); (b) W. H. Fisher, T. Carrington, S. V. Filseth, C. M. Sadowski, and C. H. Dugan, Chem. Phys. 82, 443 (1983); (c) W. H. Fisher, R. Eng. T. Carrington, C. H. Dugan, S. V. Filseth, and C. M. Sadowski, ibid. 89, 457 (1984); (d) W. J. Marinelli, N. Sivakumar, and P. L. Houston, J. Phys. Chem. 88, 6685 (1984); (e) I. Nadler, H. Reisler, and C. Wittig, Chem. Phys. Lett. 103, 451 (1984); (f) G. E. Hall, N. Sivakumar, and P. L. Houston, J. Chem. Phys. 84, 2120 (1986); (g) see also Refs. 5 and 9. (h) M. J. Sabety-Dzvonik and R. J. Cody, J. Chem. Phys. 66, 125 (1977).

"(a) W. M. Pitts and A. P. Baronavski, Chem. Phys. Lett. 71, 395 (1980); (b) W. P. Hess and S. R. Leone, J. Chem. Phys. 86, 3773 (1987).

${ }^{9}$ I. Nadler, D. Mahgerefteh, H. Reisler, and C. Wittig, J. Chem. Phys. 82, 3885 (1985)

II'(a) M. A. O'Halloran, H. Joswig, and R. N. Zare, J. Chem. Phys. 87, 303 (1987); E. Hasselbrink, J. R. Waldeck, and R. N. Zare (to be published); (b) see Ref. 7(f)

I' (a) M. D. Morse, K. F. Freed, and Y. B. Band, J. Chem. Phys. 70, 3620 (1979); (b) M. N. R. Ashfold, M. T. Macpherson, and J. P. Simons, Top. Curr. Chem. 86, 1 (1979); J. P. Simons, in Gas Kinetics and Energy Trans. fer, edited by P. G. Ashmore and R. J. Donovan (Chemical Society, London, 1977), Vol. 2, p. 58; (c) see also Ref. 9.

${ }^{12}$ E. M. Goldfield, P. L. Houston, and G. S. Ezra, J. Chem. Phys. 84, 3120 (1986).

${ }^{13}$ (a) K. E. Holdy, L. C. Klotz, and K. R. Wilson, J. Chem. Phys. 52, 4588 (1970); (b) J. A. Beswick and J. Jortner, Chem. Phys. 24, 1 (1977); (c) U. Halavee and M. Shapiro, ibid. 21, 105 (1977); (d) J. A. Beswick and W. M. Gelbart, J. Phys. Chem. 84, 3148 (1980); (e) M. D. Patengill, Chem. Phys. 78, 229 (1983); 87, 419 (1984); (f) B. A. Waite, H. Helvajian, B. I. Dunlap, and A. P. Baronavski, Chem. Phys. Lett. 111, 544 (1984); (g) see also Refs. 7(d) and 11 (a).

${ }^{14}$ N. F. Scherer, J. L. Knee, D. D. Smith, and A. H. Zewail, J. Phys. Chem. 89, 5141 (1985).

${ }^{15}$ (a) A. P. Baronavski, Chem. Phys. 66, 217 (1982); (b) see Refs. 7(b) and $7(\mathrm{c})$. 
${ }^{16}$ (a) C. H. Dugan and D. Anthony, J. Phys. Chem. 91, 3929 (1987); (b) C. H. Dugan, ibid. 92, 720 (1988).

${ }^{17}$ (a) C. H. Greene and R. N. Zare, Annu. Rev. Phys. Chem. 33, 119 (1982), and references therein; (b) R. Bersohn, Isr. J. Chem. 14, 111 (1975)

is (a) R. N. Zare, Mol. Photochem. 4, 1 (1972); (b) S. Yang and R. Bersohn, J. Chem. Phys. 61, 4400 (1974); (c) C. Jonah, ibid. 55, 1915 (1971); (d) R. Schmiedl, H. Dugan, W. Meier, and K. H. Welge, Z. Phys. A 304, 137 (1982); (e) P. Andersen, G. S. Ondrey, B. Titze, and E. W. Rothe, J. Chem. Phys. 80, 2548 (1984).

${ }^{19} \beta$ is a product of three functions; that is: $\beta=2 P_{2}(\cos \chi)$ $P_{2}(\cos \alpha) f(\langle\omega\rangle\langle\tau\rangle)$, where $\chi$ is the angle between the transition moment and the molecular axis, $\alpha$ is the angle between the recoil direction and the molecular axis, and $f=\left[1+(\langle\omega\rangle\langle\tau\rangle)^{2}\right] /\left[1+4(\langle\omega\rangle\langle\tau\rangle)^{2}\right]$ describes the effect of the parent rotation on the angular distribution. For axial recoil and prompt dissociation, $\beta=2$ for the parallel transition and $\beta=-1$ for the perpendicular transition.

20) See Ref. 7(d).

${ }^{21}$ The only adjustment made was the attenuation of the probe beam, by the translation of a variably coated neutral density filter (Al coating on thin quartz substrate). We have verified that this translation does not affect the $t=0$ location, to within our experimental resolution ( $<10 \mathrm{fs}$ ).

${ }^{22}$ (a) H. Tsubomura and T. Sakata, Chem. Phys. Lett. 21, 511 (1973); K. Fuke and S. Nagakura, J. Mol. Spectrosc. 64, 139 (1977); (b) J. R. Woodworth, T. A. Green, and C. A. Frost, J. Appl. Phys. 57, 1648 (1985).

${ }^{23}$ P. M. Felker and A. H. Zewail, Adv. Chem. Phys. 70, 265 (1988), and references therein.

${ }^{24}$ P. M. Felker and A. H. Zewail, J. Chem. Phys. 86, 2460 (1987); J. S. Baskin, P. M. Felker, and A. H. Zewail, ibid, 86, 2483 (1987).

25 (a) C. K. Luk and R. Bersohn, J. Chem. Phys. 58, 2153 (1973); (b) W. M. Jackson, ibid. 61, 4177 (1974); (c) T. J. Cook and D. H. Levy, ibid. 57, 5059 (1972).

${ }^{26}$ See, for example: (a) R. Vasudev, R. N. Zare, and R. N. Dixon, J. Chem. Phys. 80, 4863 (1984); (b) P. L. Houston, J. Phys. Chem. 91 (Special Issue of Dynamical Stereochemistry), 5388 (1987); (c) J. Simons, ibid, 91, 5378 (1987).

${ }^{27}$ (a) T. F. Heinz, S. L. Palfrey, and K. B. Eisenthal, Opt. Lett. 9, 359
(1984); S. L. Palfrey and T. F. Heinz, J. Opt. Soc. Am. B 2, 674 (1985); (b) M. W. Balk and G. R. Fleming, J. Chem. Phys. 83, 4300 (1985); (c) R. Trebino, C. E. Barker, and A. E. Siegman, IEEE J. Quantum Electron. QE-22, 1413 (1986); (d) P. C. Becker, R. L. Fork, C. H. Brito Cruz, J. P. Gordon, and C. V. Shank, Phys. Rev, Lett. 60, 2462 (1988).

${ }^{28}$ The available energy is calculated as follows: We take the total energy for the $306 \mathrm{~nm}$ excitation and subtract the bond energy $\sim 74 \mathrm{kcal} / \mathrm{mol}$ ( $\mathrm{see}$, e.g., Ref. 12 and references therein). When subtracting the average rotational energy of the $\mathrm{CN}\left(1250 \mathrm{~cm}^{-1}\right)$ from this available energy, we obtain the translational energy.

${ }^{29}$ R. Bersohn and A. H. Zewail, Ber. Bunsenges, Phys. Chem. 92, 373 (1988).

${ }^{30}$ (a) T. S. Rose, M. J. Rosker, and A. H. Zewail, J. Chem. Phys. 88, 6672 (1988); (b) M. J. Rosker, T. S. Rose, and A. H. Zewail, Chem. Phys. Lett. 146, 175 (1988).

${ }^{31}$ See Ref. 13 [e.g., (a) and (c) ] for extraction of $L^{-1}=\alpha$ from the absorption spectrum.

${ }^{32}$ We assume no van der Waals well or switching in shape between $V_{1}$ and $V_{2}$. This is discussed in detail in Ref. 33.

${ }^{33}$ R. B. Bernstein and A. H. Zewail, J. Chem. Phys. (submitted).

${ }^{34}$ See Fig. 7 of Ref. 7 (b).

${ }^{35}$ In the experiments, the probe tuning procedure had the effect of also changing the pump wavelength, in the range from 305 to $308 \mathrm{~nm}$. However, the additional energy available to the fragments as the pump frequency varied was negligible, and was not believed to affect $\tau_{1 / 2}$ significantly. Evidence for this assertion can be seen in the results obtained with a $285 \mathrm{~nm}$ pump wavelength, where $\tau_{1 / 2}$ changed only to $160 \mathrm{fs}$.

${ }^{36}$ R. Schinke, J. Phys. Chem. 92, 3195 (1988), and references therein.

${ }^{37}$ A. H. Zewail, Faraday Discuss. (to be published).

${ }^{38}$ Normalizing the $A_{\|}$and $A_{1}$ signals on an absolute scale is experimentally difficult because of delicate changes in the optical alignment when rotating the half-wave plate. We use the long time asymptote as a marker to see the change in the time dependence of the signals. In future experiments, we will attempt to normalize the data more accurately and to use magic angle methods. (See Ref. 24.)

${ }^{39} \mathrm{~K}$. Wilson (private communication).

${ }^{40}$ S. O. Williams and D. G. Imre, J. Phys. Chem. (in press). 